\title{
Spontaneous ovarian hyperstimulation syndrome in a young female subject with a lingual thyroid and primary hypothyroidism
}

Soo Jeong Kim, Jee Hee Yoon, Hee Kyung Kim, and Ho-Cheol Kang

Department of Internal Medicine, Chonnam National University Hwasun Hospital, Hwasun, Korea

Received: November 17, 2015

Revised : March 29, 2016

Accepted: May 15, 2016

\section{Correspondence to}

Ho-Cheol Kang M.D.

Department of Internal Medicine, Chonnam National University

Hwasun Hospital, 322 Seoyang-ro, Hwasun 58128, Korea

Tel: $+82-61-379-7620$

Fax: $+82-61-379-7628$

E-mail:drkang@chonnam.ac.kr
To the Editor,

Ovarian hyperstimulation syndrome (OHSS) is a potentially life-threatening condition that classically occurs in adult women undergoing the excessive administration of gonadotropins as an assisted reproductive technique to induce ovulation [1]. In the absence of stimulation from exogenous gonadotropins, rare cases of spontaneous OHSS have been reported in women with severe primary hypothyroidism, polycystic ovary syndrome while pregnant, gonadotroph pituitary adenoma, and normal pregnancy [2-4]. In women with primary hypothyroidism, extremely elevated levels of thyroid stimulating hormone
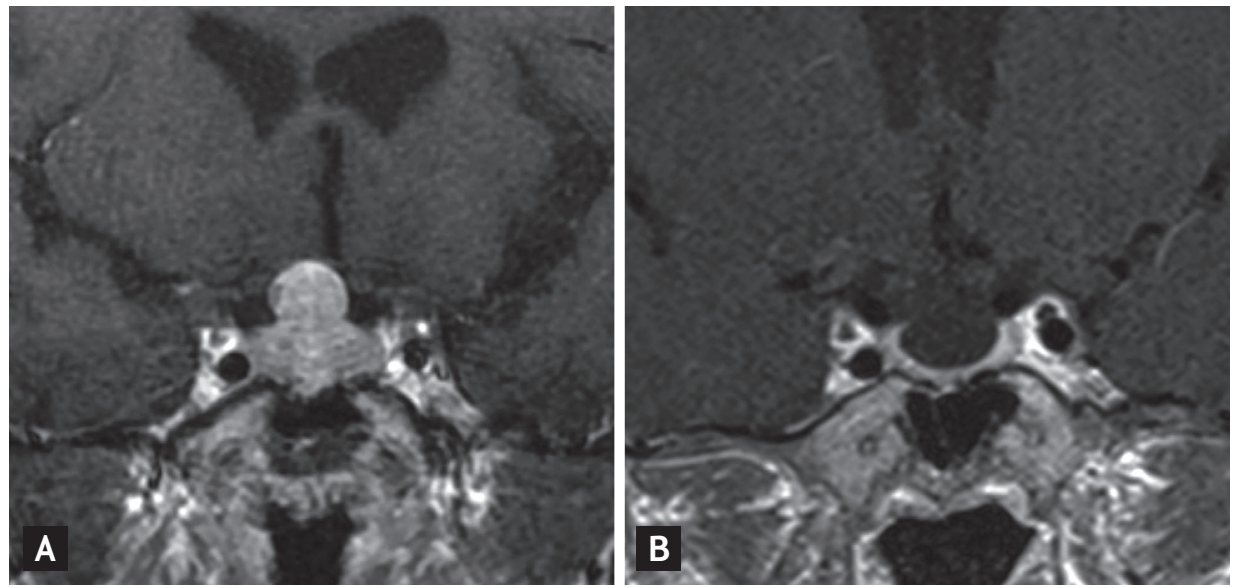

Figure 1. (A) T1-gadolinium-enhanced coronal magnetic resonance imaging (MRI) scan showing diffuse pituitary enlargement without a definite focal mass. (B) Follow-up MRI scan 6 months after levothyroxine therapy revealing a marked regression of the pituitary enlargement. 
to the parents' concern regarding the patient's poor academic performance. The patient had no significant medical history, was not taking any medications, weighed 45 $\mathrm{kg}$, and was $127 \mathrm{~cm}$ in height; according to the Korean growth curve for young females (2 to 20 years of age), her height was below the third percentile. The patient's menarche was at 10 years of age, after which she experienced irregular menses with periods of amenorrhea that sometimes lasted for 4 to 5 months. The clinical signs and symptoms of hypothyroidism were not conspicuous, but she exhibited very slow responses to questions, and her face was round and puffy. There was no enlargement of the thyroid, and a formal IQ test was not performed.

The routine biochemical tests did not reveal any abnormalities and her serum-free thyroxine and total triiodothyronine levels were $0.118 \mathrm{ng} / \mathrm{dL}$ (reference range, 0.6 to 1.7 ) and $<0.195 \mathrm{ng} / \mathrm{mL}$ (reference range, 0.8 to 1.71), respectively. However, the patient's TSH level was markedly elevated (> 1,000 $\mu \mathrm{U} / \mathrm{mL}$; reference range, 0.4 to 4.8 ), and no thyroid autoantibodies were detected. Her serum level of luteinizing hormone ( $\mathrm{LH})$ was undetectable but her serum level of follicle stimulating hormone (FSH; $7.31 \mathrm{mIU} / \mathrm{mL}$ ) was within a normal range; the reference ranges for FSH and LH in the follicular phase
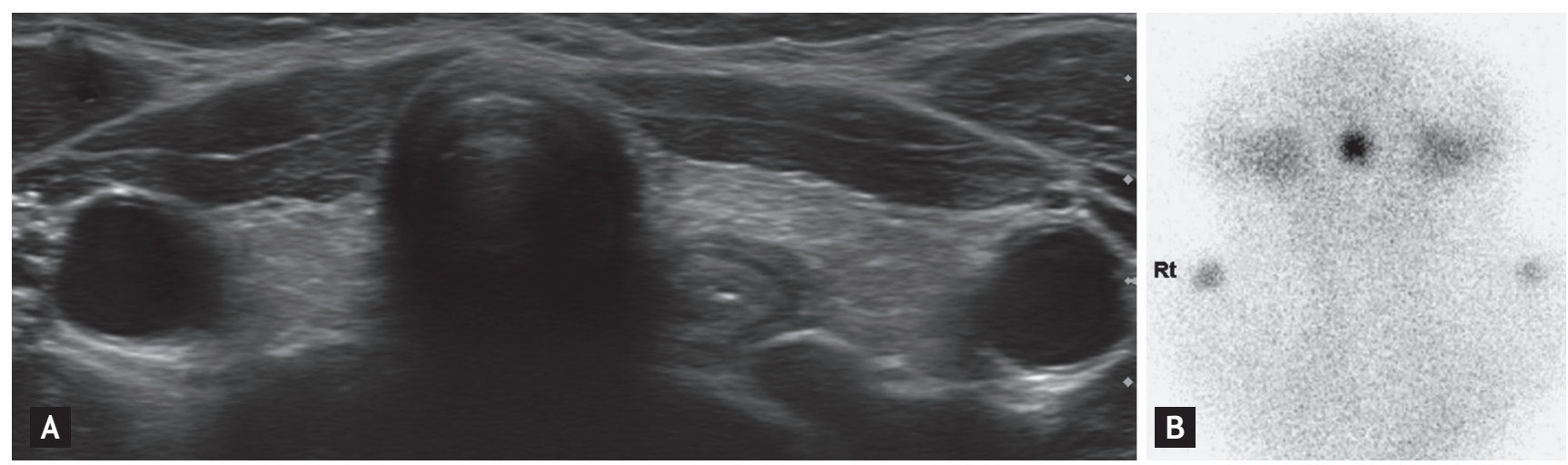

Figure 2. (A) A neck ultrasonography showing that the thyroid bed was replaced by fat with no identifiable thyroid tissue. (B) Technetium-99m pertechnetate scan showing a localized ectopic lingual thyroid gland. Rt, right.
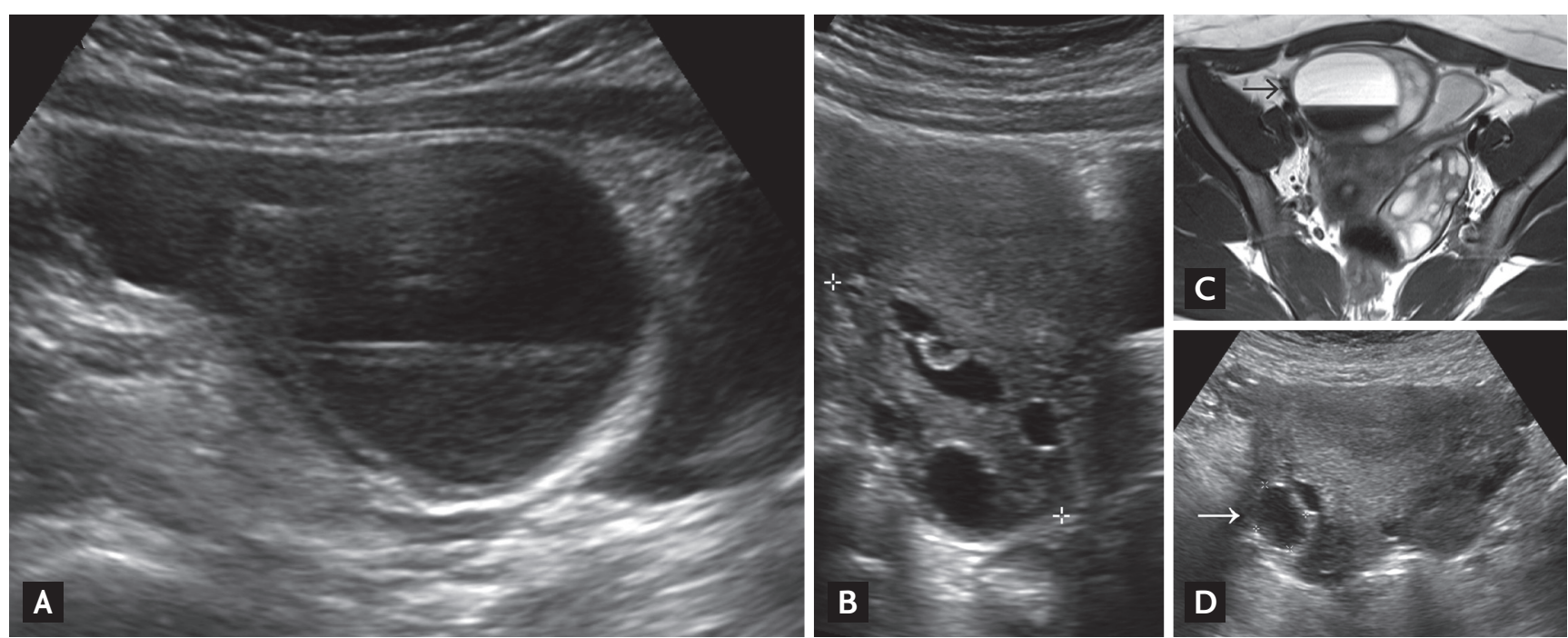

Figure 3. (A, B) Abdominal ultrasonography (US) showing massive cystic enlargement in both ovaries (A, right; B, left); a large cyst with fluid-hemorrhage level is visible in the right ovary. (C) Pelvic magnetic resonance imaging scan showing diffuse enlargement of both ovaries and a right ovarian mass with a cystic component that measured as $5.2 \times 4.3 \times 5.2 \mathrm{~cm}(\mathrm{arrow})$. (D) The marked regression of the hemorrhagic cyst and bilateral ovarian enlargement was evident in follow-up abdominal US scans 3 months after the initiation of levothyroxine therapy; right ovary: $2.0 \mathrm{~cm}$ (white arrow). 
are 1.1 to 9.6 and 0.8 to $25.8 \mathrm{mIU} / \mathrm{mL}$, respectively. The levels of her other pituitary hormones were as follows: growth hormone $(0.55 \mathrm{ng} / \mathrm{mL})$, insulin-like growth factor $1(104.67 \mathrm{ng} / \mathrm{mL})$, prolactin $(46.10 \mathrm{ng} / \mathrm{mL})$, adrenocorticotropic hormone $(16 \mathrm{pg} / \mathrm{mL})$, and cortisol $(10.0 \mu \mathrm{g} / \mathrm{mL})$.

A neck ultrasonography (US) scan did not show any thyroid tissue in a eutopic position and revealed that the thyroid bed was replaced with fatty tissue (Fig. 2A). However, a subsequent thyroid scan showed an ectopic lingual thyroid gland (Fig. 2B). An abdominal US was performed because the patient complained of mild abdominal discomfort and nausea. She had an enlargement of both ovaries (right, $8.9 \times 4.6 \mathrm{~cm}$; left, $5.9 \times 8.2 \mathrm{~cm}$ ) with multiple cystic changes, and an abdominal US scan unexpectedly revealed a large hemorrhagic cyst in the right ovary (the largest diameter was $67 \mathrm{~mm}$ ) (Fig. 3A and 3B). A pelvic $M R I$ scan showed the hemorrhagic cyst in the right ovary to be $5.2 \times 4.3 \times 5.2 \mathrm{~cm}$ in size and that both ovaries and the uterus were enlarged compared with a control group of a similar age (Fig. ${ }_{3} \mathrm{C}$ ). The patient's serum level of cancer antigen 125 was normal $(25.87 \mathrm{U} / \mathrm{mL}$; range, o to 35 ), and her total human chorionic gonadotropin level was undetectable.

The patient was diagnosed with primary hypothyroidism (which stemmed from the ectopic lingual thyroid), bilateral ovarian cystic enlargement (which was similar to mild OHSS), and reactive pituitary hyperplasia. Subsequently, levothyroxine (LT4; $100 \mu \mathrm{g} /$ day) therapy was initiated, and an abdominal US scan revealed significant reductions in the sizes of both ovaries 1 month after the beginning of $\mathrm{LT}_{4}$ treatment. At this time, the patient's serum TSH level was $0.841 \mu \mathrm{U} / \mathrm{mL}$, and it has since remained within a normal range. At 3 months after $\mathrm{LT}_{4}$ treatment was initiated, both ovaries had returned to a normal size and appearance with a complete regression of the ovarian cyst (Fig. 3D). At 6 months, the patient's menstrual cycle had normalized and a follow-up sellar MRI scan revealed a marked reduction in the size of the pituitary gland (Fig. $1 \mathrm{~B}$ ). The patient underwent a $7.8 \mathrm{~cm}$ growth spurt over 12 months following the achievement of euthyroidism.

The present report describes the case of a young female patient with primary hypothyroidism complicated by reactive thyrotroph hyperplasia and spontaneous OHSS that mimicked ovarian tumors. $\mathrm{LT}_{4}$ replacement reduced the ovarian volume and resulted in a regression of the ovarian cysts which enabled the differentiation of OHSS from the presence of primary ovarian tumors without costly or invasive diagnostic procedures.

Using serial US scans, the regression of the ovarian cysts and a reduction in ovarian volume was observed 3 months after the initiation of $\mathrm{LT}_{4}$ replacement. Other studies investigating non-pregnant hypothyroid patients found a considerable regression of cysts after 3 to 4 months of treatment for the hypothyroidism with $100 \mu \mathrm{g}$ doses of $\mathrm{LT}_{4}$ [3]. It is noteworthy that the kinetics of the declining serum levels of TSH are closely related to the regression of the ovarian cysts; this temporal correlation supports the suggestion that the elevated TSH mimicked endogenous gonadotropins and caused ovarian hyperstimulation.

Primary hypothyroidism and OHSS are likely linked by the stimulation of the ovaries following extreme elevations in the level of TSH because gonadotropins and TSH share the same $\alpha$ subunit. Additionally, TSH has weak FSH- and LH-like action at the FSH and LH receptors, which can cause gonadal stimulation. This hypothesis would explain why the present patient had relatively low FSH and LH levels. In the present case and in most previously reported cases, TSH-induced OHSS occurred in relatively young females; thus, it is possible that immature ovaries are more vulnerable to excessive levels of TSH [3].

The diffuse enlargement of the pituitary gland in the present patient was thought to be due to compensatory thyrotroph hyperplasia. In primary hypothyroidism, an attenuation of negative feedback signals from peripheral thyroid hormones induces thyrotroph and lactotroph cell hyperplasia and results in excessive levels of TSH and prolactin [5]. In the present case, the patient's compensatory pituitary hyperplasia might have been mistakenly diagnosed as a pituitary macroadenoma, but the marked regression of the pituitary enlargement after $\mathrm{LT}_{4}$ replacement allowed for a differential diagnosis.

The present report describes the resolution of ovarian cysts and the normalization of ovarian size in a young female patient with a lingual thyroid following $\mathrm{LT}_{4}$ administration. To avoid costly interventions, a diagnosis of OHSS should be considered when there is bilateral ovarian enlargement in the clinical context of profound primary hypothyroidism. 
Keywords: Hypothyroidism; Spontaneous ovarian hyperstimulation syndrome; Pituitary hyperplasia

\section{Conflict of interest}

No potential conflict of interest relevant to this article was reported.

\section{REFERENCES}

1. Braat DD, Schutte JM, Bernardus RE, Mooij TM, van Leeuwen FE. Maternal death related to IVF in the Netherlands 1984-2008. Hum Reprod 2010;25:1782-1786.

2. Halupczok J, Bidzinska-Speichert B, Lenarcik-Kabza A, Zielinski G, Filus A, Maksymowicz M. Gonadotroph adenoma causing ovarian hyperstimulation syndrome in a premenopausal woman. Gynecol Endocrinol 2014;30:774777.

3. Langroudi RM, Amlashi FG, Emami MH. Ovarian cyst regression with levothyroxine in ovarian hyperstimulation syndrome associated with hypothyroidism. Endocrinol Diabetes Metab Case Rep 2013;2013:130006.

4. Zalel Y, Katz Z, Caspi B, Ben-Hur H, Dgani R, Insler V. Spontaneous ovarian hyperstimulation syndrome concomitant with spontaneous pregnancy in a woman with polycystic ovary disease. Am J Obstet Gynecol 1992;167:122124.

5. Sarlis NJ, Brucker-Davis F, Doppman JL, Skarulis MC. MRI-demonstrable regression of a pituitary mass in a case of primary hypothyroidism after a week of acute thyroid hormone therapy. J Clin Endocrinol Metab 1997;82:808811. 